\title{
COMPARING THREE DIFFERENT SURGICAL GUIDES PROTOCOL ON THE MARGINAL BONE LOSS AROUND IMPLANTS IN IMPLANT ASSISTED MANDIBULAR OVERDENTURE
}

\author{
Mahmoud El Moutassim-Bellah El Homossany* and Ahmed Mostafa Abdelfattah ${ }^{* *}$
}

\begin{abstract}
Aim of the study: This study was conducted to assess the efficacy of different guided surgery protocols on mean marginal bone loss around implants in implant assisted mandibular over denture.

Materials and methods: Twenty-one edentulous patients were selected for this study. They were randomly divided into three different groups. In the first group implants were placed by free hand technique. In the second group partial limiting surgical guide was used; the surgical guide was used for pilot drilling. In the third group completely limiting surgical guide was used; the surgical guide was used for the whole drilling sequence. Mean marginal bone loss was measured using CBCT at 6 months, 12month and 18 months interval. Results were collected and tabulated for statistical analysis.
\end{abstract}

Results: One-way ANOVA test showed no statistical significance between the three groups in all time intervals.

Conclusion: Within the limitation of this study, no statistically significant differences could be found between the guided groups and the free hand group at the 18 months follow-up period.

\section{INTRODUCTION}

Osseointegrated implants are a practical alternative to traditional prosthodontics. They present a treatment option to improve stability and retention of complete dentures. However, designing an implant-supported prosthesis with function and aesthetics is a challenge. ${ }^{(1)}$

Pre surgical planning is essential to achieve excellent aesthetic and functional outcomes with dental implants. Guided implant placement also helps to reduce treatment time, surgical error, patient apprehension in addition to accurate dental implant placement. ${ }^{(2)}$

Practitioners have generally used conventional dental radiographs (periapical and panoramic) in addition to conventionally fabricated surgical guides. However, Surgical guides conventionally fabricated on diagnostic stone casts do not provide 
information about the varying thicknesses of the mucosa, topography of the underlying bone, or anatomical structures furthermore, they do not remain stable during surgery. ${ }^{(3)}$

However with the usage of Cone beam CT (computerized tomography) (CBCT) and implant designing computer software programs for pre surgical implant planning, practitioners can now simulate ideal implant placement and treatment planning. In addition, prosthetically directed implant placement using 3D printed surgical guides based on pre surgical planning can ensure precise implant placement and predictable prosthetic outcomes. ${ }^{(4)}$

The design of the surgical guide can be classified into non limiting, partially limiting and completely limiting according to the amount of surgical restriction offered by the surgical guide design. In partial limiting surgical guides only pilot drilling is made through the guide. However, in completely limiting surgical guides the whole drilling sequence is made through the guide. ${ }^{(5)}$

\section{Methodology}

The study population in this study was composed of 21 completely edentulous patients attending the Removable Prosthodontics Department clinic, Faculty of Dentistry Ain shams University. They were selected according to the following criteria: non-smokers, 50-70 years in age, free from systemic diseases that may affect osseointegration as uncontrolled diabetes mellitus, good oral hygiene and motivation. Intra oral examination was performed to ensure presence of keratinized mucosa, absence of any lesions as well as consistency and rate of salivary secretions.

All patients received maxillary and mandibular complete dentures. A radiographic template using self cured acrylic rein and barium sulphate was made for each patient, by duplicating the denture. CT scan was made for each patient while wearing the radiographic template for diagnosis and planning of implant placement.
Randomization was performed by a randomization table provided by statistician. The randomization of patients was made using a computerized program (statistical package for social sciences) (SPSS). The number of cases included in this study was randomly allocated into three groups.

The randomized patients were equally divided into 3 groups; 7 patients in each group. In Group I the implants were planned to be placed free hand, in group II partial limiting surgical guide was planned to be used for pilot drilling of the implants and in group III a completely limiting surgical guide was planned to be used.

In group I the denture duplicate was used to mark the position of the implants. During the surgery, a hole was made in the denture duplicate at the desired implant position and then seated in place. A mark was then made intraorally opposite the hole. A flap was raised and the labial flange of the duplicate opposite to the flap was removed, then osteotomy drilling started.

The surgical guide was designed for patients in group II and III to be partial limiting and complete limiting respectively and then printed. For fabrication of surgical guides, the DICOM files of the cone beam CT were exported into the implant design software, the implant position and angulation were designed and position of the fixation pins was determined (three pins, one in the midline and one posterior on each side). The guiding sleeve was then planned in each side. Extension of the guide was determined. The design of the surgical guide was converted to a STL format on the same software and exported for $3 \mathrm{D}$ printing using a $3 \mathrm{D}$ printer. The template was fabricated from a photo-polymerized methacrylate resin.

During surgery, the surgical guide was placed in position and stabilized against the upper denture by using the centric occluding relation record for correct stabilization .Three osteotomies corresponding to the position of the fixation pins were made (one 
near the midline of the mandible, one on the right buccal side posteriorly and one on the left buccal side posteriorly) to secure the guided stent in place.

Each patient in each group received 2 endosteal interforaminal implant to retain the mandibular denture. Surgery was performed under local anathesia (Mepiccaine local anathesia, Alexandria company for pharmaceuticals and chemical industry). Implant diameter ranged from 3.5$3.75-4.2 \mathrm{~mm}$ and for implant length it ranged from 10-12mm(Reactive, Implant Direct, USA). Intermittent drilling under copious irrigation using sharp drills was made to reduce heat generation.

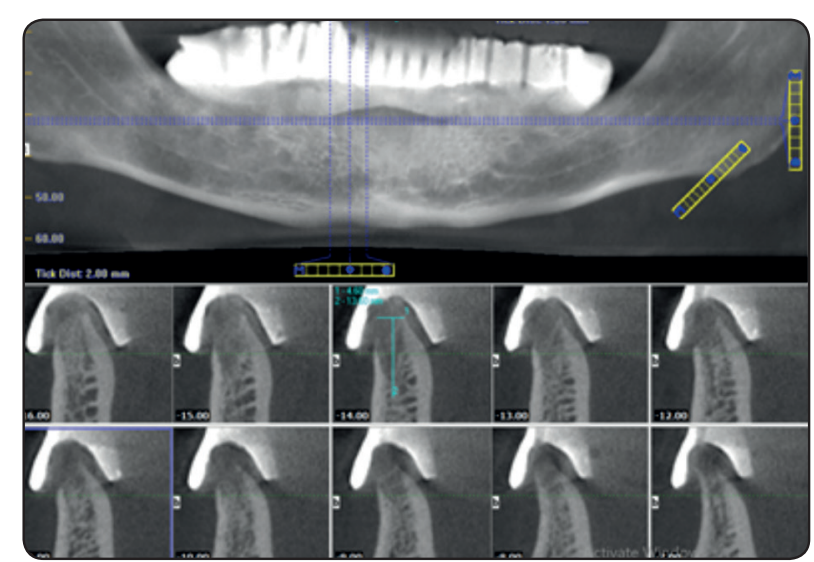

Fig. (1) Preoperative CBCT using radiographic template
For group II and III external refrigerated saline was also used for further irrigation to control further heat generation. Following implant placement by 3 months, conventional loading protocol was adopted. Ball abutments were secured to the implants and housings were picked up in the lower denture. The mean crestal bone loss for each implant was measured using cone beam CT(K.V.P 85, M.A 16, resolution 100-150 voxel, F.O.V 7*14.5*14.5 and scanning time $20 \mathrm{Sec}$ ) at the time of 6,12 , and 18 months interval. The mean marginal bone loss was the mean of the marginal bone loss on the mesial, distal, buccal and lingual sides of the implant. The amount of bone loss was calculated from the implant platform to the level of bone crest in $\mathrm{mm}$ on each side of the implant using the Cone beam CT software ruler.

\section{Statistical analysis:}

Recorded data were analyzed using the statistical package for social sciences, version 20.0 (SPSS Inc., Chicago, Illinois, USA). Quantitative data were expressed as mean \pm standard deviation (SD).

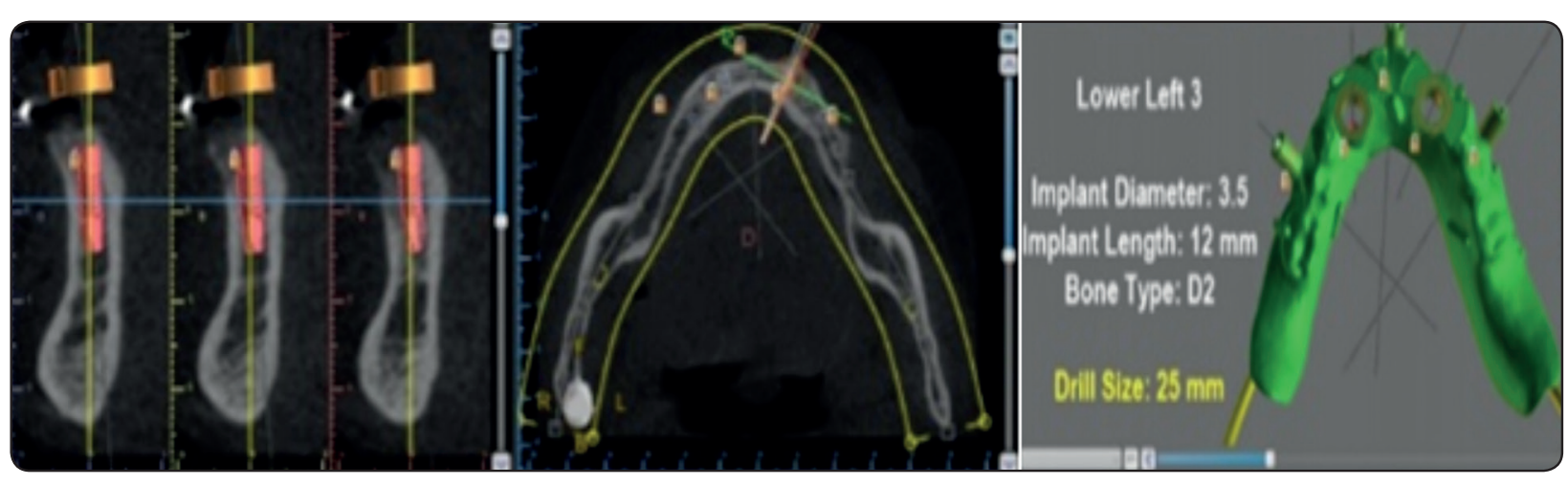

Fig. (2) Planning the implant sites and designing the surgical guide for the guided groups (II andIII) 

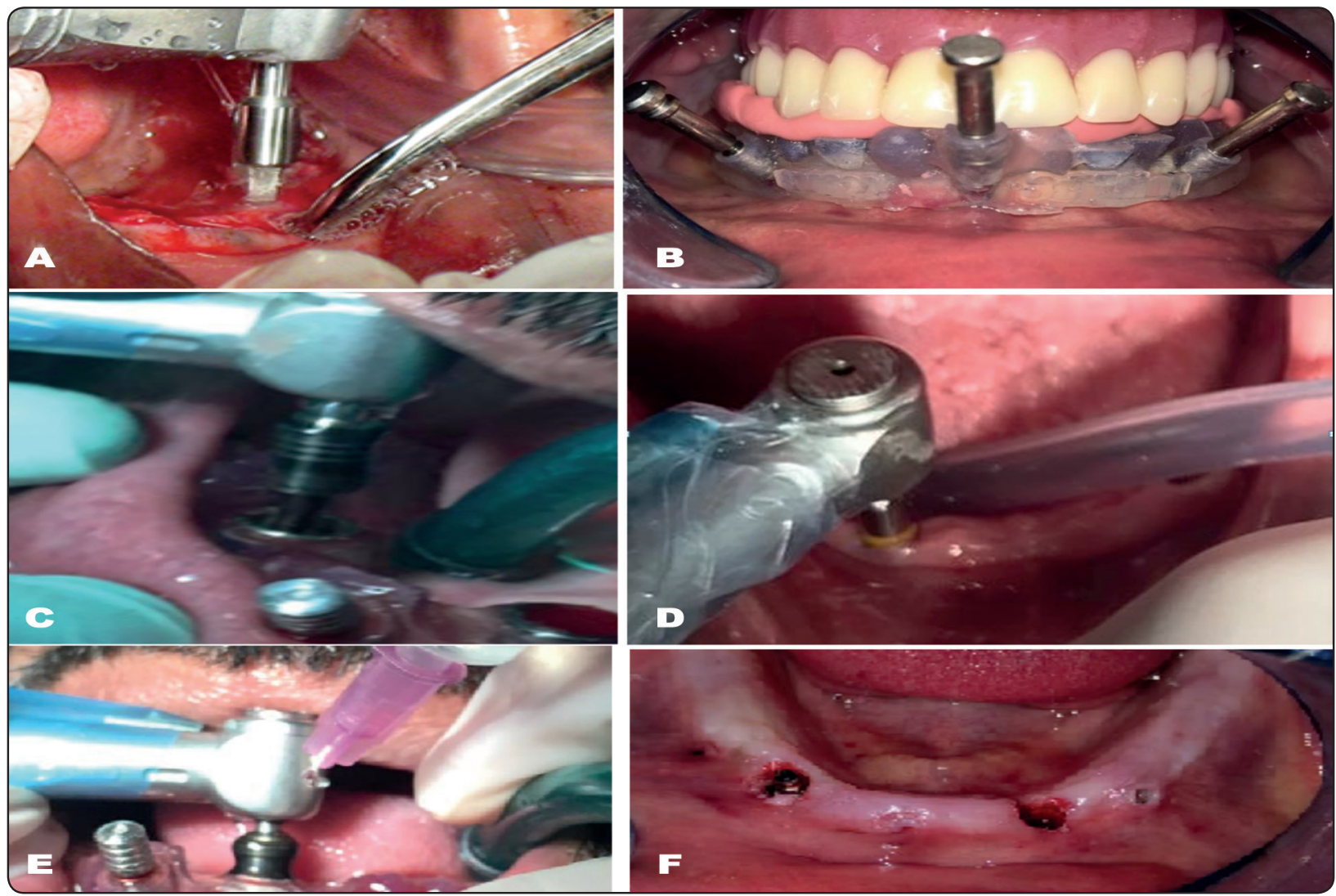

Fig. (3) A: preparing the osteotomy site after flap reflection in group I. B: stabilization of the surgical guide against the upper denture in groups II and III. C: Drilling through the guide using pilot drill in group II. D: sequential drilling continued free hand in group II. E: Drilling done till the final drill through the guide in group III. F: Implants inserted in their position
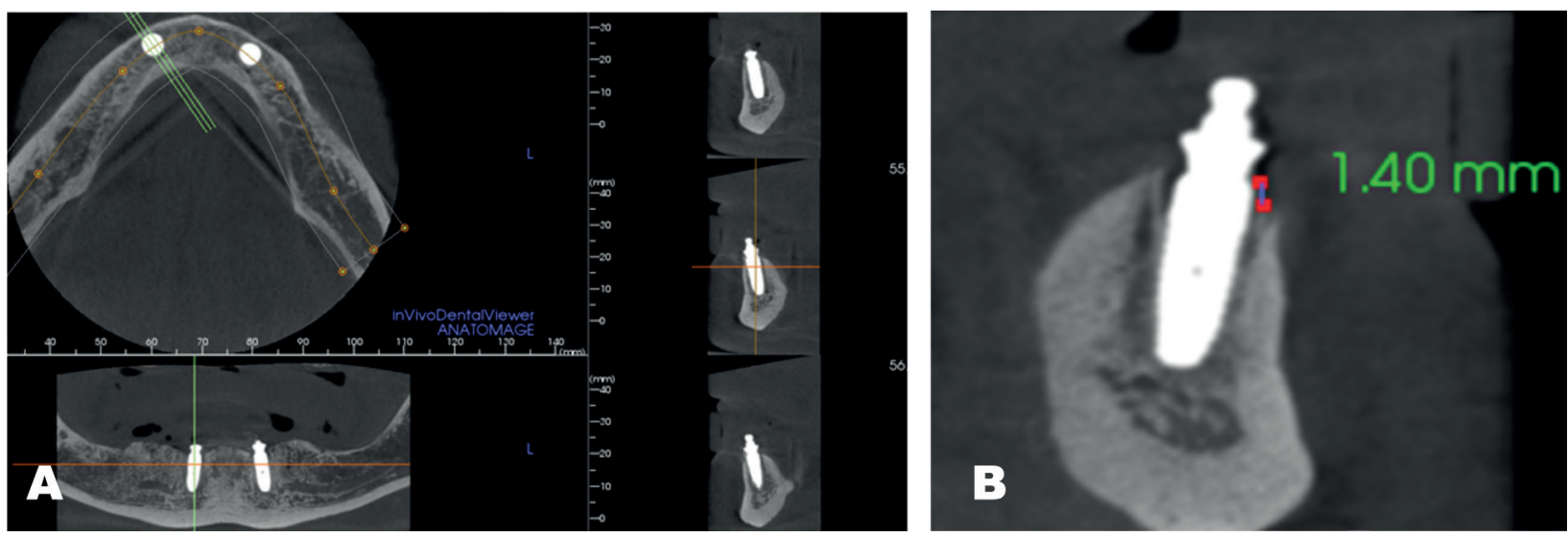

Fig. (4) A: Postoperative CBCT - B: Measuring the marginal bone loss using the CBCT software ruler 
TABLE (1): Comparison between the three groups according to mean marginal bone loss at $6,12,18$ months interval.

\begin{tabular}{|l|c|c|c|c|c|}
\hline Mean marginal bone loss (mm) & $\begin{array}{c}\text { Free Hand } \\
(\boldsymbol{n}=\mathbf{1 4})\end{array}$ & $\begin{array}{c}\text { Fully Guided } \\
(\boldsymbol{n}=\mathbf{1 4})\end{array}$ & $\begin{array}{c}\text { Partially Guided } \\
(\boldsymbol{n}=\mathbf{1 4})\end{array}$ & ANOVA & p-value \\
\hline $\begin{array}{l}\mathbf{6} \text { months } \\
\text { Mean } \pm \mathrm{SD}\end{array}$ & $0.46 \pm 0.09$ & $0.49 \pm 0.10$ & $0.49 \pm 0.09$ & 0.541 & 0.587 \\
\hline $\begin{array}{l}\mathbf{1 2} \text { months } \\
\text { Mean } \pm \mathrm{SD}\end{array}$ & $0.82 \pm 0.14$ & $0.89 \pm 0.14$ & $0.81 \pm 0.14$ & 1.31 & 0.281 \\
\hline $\begin{array}{l}\mathbf{1 8} \text { months } \\
\text { Mean } \pm \mathrm{SD}\end{array}$ & $1.14 \pm 0.17$ & $1.21 \pm 0.17$ & $1.11 \pm 0.19$ & 1.196 & 0.313 \\
\hline
\end{tabular}

\section{RESULTS}

The mean marginal bone loss for group I at six months interval was $0.46 \mathrm{~mm}( \pm 0.09)$ and for group II was $0.49 \mathrm{~mm}( \pm 0.09)$ and for group III was $0.49 \mathrm{~mm}( \pm 0.1)$. However for twelve month interval was $0.82 \mathrm{~mm}( \pm 0.14)$ for group I , $0.81 \mathrm{~mm}$ $( \pm 0.14)$ for group II and $0.89 \mathrm{~mm}( \pm 0.14)$ for group III. At eighteen month interval, the mean marginal bone loss was $1.14 \mathrm{~mm}( \pm 0.17)$ for group I and $1.11 \mathrm{~mm}( \pm 0.19)$ for group II and for group III, it was $1.21 \mathrm{~mm}( \pm 0.17)$.

\section{The following tests were done:}

- A one-way analysis of variance (ANOVA) test when comparing between more than two means.

- The confidence interval was set to $95 \%$ and the margin of error accepted was set to $5 \%$. the p-value was considered significant as the following:

- P-value $\leq 0.05$ was considered significant.

- P-value $\leq 0.001$ was considered as highly significant.

- P-value >0.05 was considered insignificant.

The results of the present study are demonstrated in the following tables and figures.

This table shows no statistically significant difference between the three groups according to mean crestal bone loss at $6,12,18$ months interval.

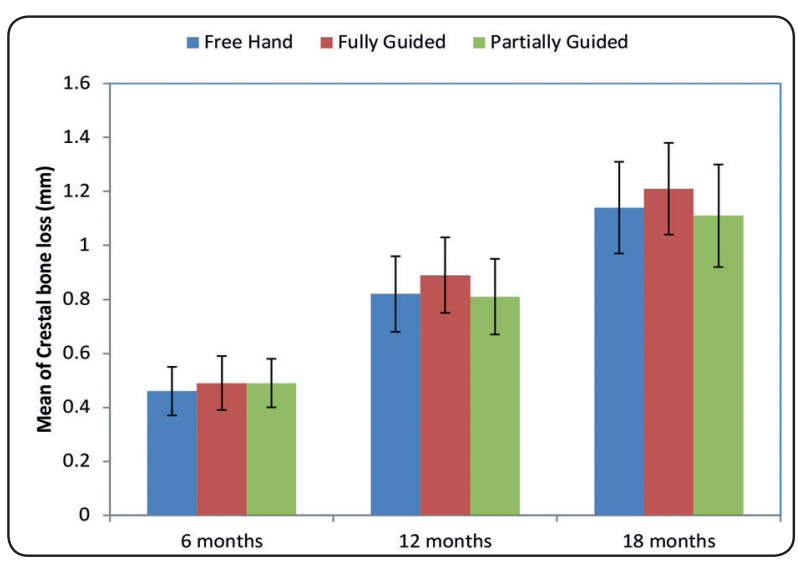

Fig. (5): Error bar between the three groups according to mean marginal bone loss at $6,12,18$ months interval.

\section{DISCUSSION}

Maligned implants often complicate the clinical laboratory procedures employed for fabrication of superstructures. Due to improper load distribution, an overall increase in stress concentration on supporting structures may occur. This may compromise the maintenance of the bone implant interface. Excellent outcomes with dental implants can be achieved by guided implant placement. Inaccurate positioning of dental implants can cause aesthetic, biologic and prosthetic complications as high rates of crestal bone loss, screw loosening and fracture of prosthetic components. ${ }^{(6)}$

The advent of cone beam computed tomography (CBCT) has played a role in the development of guided surgery techniques. ${ }^{(7)}$ 
Cone beam CT (CBCT) coupled with implant design software has been introduced for pre surgical implant planning. The use of computer-aided design/computer-aided manufacturing (CAD/CAM) stereolithographic (SLA) surgical guides also offer a significant advantage to the surgeon by improving precision and predictability of implant placement. Moreover, complications such as mandibular nerve damage, sinus perforation, fenestrations, and dehiscence are minimized. ${ }^{(8)}$

A systematic review of various studies has found that the implant cumulative survival rate for guided implant placement is comparable to non-guided one. ${ }^{(9)}$.

From a heat generation standpoint, preparing an implant site with the surgical guide produces more heat generation than non guided implant site preparation. The use of surgical guides was found to increase the temperature in the osteotomy site during drilling when compared to non guided placement as it blocks the coolant from reaching the osteotomy site effectively. High temperature during osteotomy site preparation causes bone necrosis and affects implant osseointegration. ${ }^{(10,11)}$

Based on theoretical analysis and Finite Element Analysis simulation, the temperature distribution of the drilling area in the placement of dental implants under surgical guide was determined at average depth of $8 \mathrm{~mm}$. $^{(12)}$

The mean maximum temperatures were the highest (about $39.8{ }^{\circ} \mathrm{C}$ ) was found at a depth of 9 $\mathrm{mm}$ in another study ${ }^{(13)}$.On the other hand, a study did not find a significant difference in the mean maximum temperature generated in the osteotomy sites prepared by surgical guides or free hand..$^{(14,15)}$

Refrigerated saline and external irrigation were used during drilling to decrease the temperature within the osteotomy site in group II and III. Further precautions were taken in both groups as intermittent drilling, decreased drill speed, sharp drills and regular withdrawal of the drill.
Delayed loading was adopted as the rates of implant loss when using immediate loading with ball attachments are higher. ${ }^{(16)}$

No implant loss was reported during the follow up period. This matches the results presented by various studies which found that the implant cumulative survival rate for guided implant placement is comparable to non-guided one..$^{(9)}$

In a recent systematic review of guided implant placement in the maxilla, all studies reported survival rates of $97.8 \%$ or higher after 1 year ${ }^{(17)}$ .Implants placed with a guide appear to have a survival rate comparable with those placed without a guide with 1-year survival rates of $94 \%$. $^{(18)}$

This high survival rate is consistent with similar clinical trials, ${ }^{(19,20)}$ which may have been because of strict inclusion and exclusion criteria, together with comprehensive diagnosis and screening.

It was reported that small changes in horizontal bone height may not be detectable on the panoramic radiograph due to low resolution. In addition, the degree of magnification has been reported not to be uniform across the same panoramic survey ${ }^{(21,22)}$ Therefore, Cone Beam CT was used in this study for the evaluation of marginal bone loss.

The null hypothesis that no difference in mean marginal bone loss to be found between the groups was accepted.

Marginal bone loss was evaluated using the implant platform as a reference in relation to the alveolar bone crest using CBCT at 6,12 and 18 months interval. The progression and amount of marginal bone loss around the implants are important data for the diagnosis of peri-implant health. The average mean bone loss in our study in the three groups is within the range defined in an another study. ${ }^{(23)}$

A randomized controlled clinical trial comparing guided with nonguided implant placement at a 3-year follow-up was made. The results of this trial are matching our study results; the mean marginal bone loss for 12 month period was ranging from 
(1.1-1.3) mm for the guided group and (1.4-1.3 mm) for the non guided one showing no statistical significance . ${ }^{(24)}$

The present trial is consistent with the results of a systematic review about guided implant surgery. No significant difference of peri-implant bone loss between guided and non guided groups at 1-year follow-up or up to 3-year follow up was detected. ${ }^{(17)}$

A study on full-Mouth Rehabilitation about implants inserted with computer-guided flapless surgery showed that the implant survival rate was $97.9 \%$, whereas the average marginal bone loss was $1.9 \mathrm{~mm}( \pm 1.3)$ after 3 years. ${ }^{(25)}$

Another study measuring marginal bone changes around implants inserted in edentulous jaws following computer guided treatment planning and flapless surgery found out that the mean marginal bone change was $1.2 \mathrm{~mm}( \pm 1.4){ }^{(26)}$

\section{CONCLUSION}

Within the limitation of this study, no statistically significant differences could be found between the guided groups and the free hand group at the 18 months follow-up period.

\section{REFERENCES}

1. Niedermaier R., Stelzle F., Riemann M., Bolz W., Schuh P. and Wachtel H. Implant-Supported Immediately Loaded Fixed Full-Arch Dentures: Evaluation of Implant Survival Rates in a Case Cohort of up to 7 Years. J Clin Impl Dent and Related Res, 2017;19: 4-19

2. Alevizakos V, Mitov G, Stoetzer M and Von See C. A retrospective study of accuracy of template guided versus free hand implant placement: A non-radiologic method. J Oral Maxillofac Surg,2019;128:220-6.

3. Jordi Gargallo-Albiol, Shayan Barootchi, Oscar SalomóColl, Hom-lay Wang,Advantages and disadvantages of implant navigation surgery. A systematic review, Annals of Anatomy - Anatomischer Anzeiger,2019; 225:1-10

4. Vermeulen J. The accuracy of implant placement by experienced surgeons: guided vs freehand approach in a simulated plastic model. Int J Oral Maxillofac Implants 2017;32:617
5. Vercruyseen M, Coucke W, Naert I, Jacobs R, Teughels W and Quirynen M. Depth and lateral deviations in guided implant surgery: an RCT comparing guided surgery with mental navigation or the use of a pilot drill template. J Clin Oral Implants Res2015;26:1315-20.

6. Chackartchi T, Romanos GE and Sculean A. Soft tissuerelated complications and management around dental implants. J Periodontol 2000. 2019; 81: 124- 38.

7. Jia P, Yang G, Hu W, Chung KH, Zhao Y, Liu M et al. Comparison of in Situ Cone Beam Computed Tomography Scan Data with Ex Vivo Optical Scan Data in the Measurement of Root Surface Area. Oral Surg, Oral Med, Oral Pathol, and Oral Radiol 2019; 128: 552-7.

8. Majzoub J, Ravida A, Starch-Jensen T, Tattan M and Suárez-López Del Amo F. The Influence of Different Grafting Materials on Alveolar Ridge Preservation: a Systematic Review. J Oral Maxillofac Res. 2019 Sep 5;10(3):e6.

9. Moraschini V, Vellosa G, Luz D and Barboza EP. Implant survival rates, marginal bone level changes and complications in full mouth rehabilitation with flapless computer guided surgery: a systematic review and meta analysis. Int J Oral Maxillofac Surg2015;44:892-901.

10- Nilay E, Alkan A, Ilday S and Bengu E. Improved Dental Implant Drill Durability and Performance Using Heat and Wear Resistant Protective Coatings, J Oral Implantology. 2018;44:168-75.

11. Montero-Aguilar A, Sibaja-Ruiz L, Avendaño E, PereiraReyes R, Pozos-Guillén A and Chavarria-Bolaños D. Evaluation of Surgical Bur Deformation and Bone Surface Roughness after Multiple Uses. J Oral and Maxillofac Surg2019; 77:1894-903.

12. Liu Y, Wu J, Zhang J, Peng W and Liao W. Numerical and Experimental Analyses on the Temperature Distribution in the Dental Implant Preparation Area when Using a Surgical Guide. J of Prosthodontics 2018; 27: 42-51.

13. Kyung Chul O, Ji-Man P, June-Sung S, Jee-Hwan K, JongEun K and Jang-Hyun K. Assessment of metal sleeve-free 3D-printed implant surgical guides. J Dent Mat 2019;35: 468-76.

14. Thaisa TO and Andréa CR .Fabrication of dental implants by the additive manufacturing method: A systematic review. J Prosth Dent 2019;122 : 270-4.

15. Yafi Firas A, Brittany C, and Al-Sabbagh M. Is Digital Guided Implant Surgery Accurate and Reliable? Dental Clinics of North America 2019;63:381-97. 
16. Ravidà A, Barootchi S, Askar H, Suárez López del Amo, Fernando \& Tavelli, Lorenzo \& Wang, Hom-Lay LongTerm Effectiveness of Extra-Short ( $\leq 6 \mathrm{~mm}$ ) Dental Implants: A Systematic Review . Int J Oral \& Maxillofac Implants. 2019;34:68-84.

17. Laleman I, Bernard L, Vercruyssen M, Jacobs R, Bornstein MM and Quirynen M. Guided implant surgery in the edentulous maxilla: a systematic review. Int J Oral Maxillofac Implants 2016;31:103-17.

18. KimWH., Lee J C., LimD., Heo Y. K., Song E S., LimY $\mathrm{J}$ and KimB. Optimized Dental Implant Fixture Design for the Desirable Stress Distribution in the Surrounding Bone Region: A Biomechanical Analysis. J. Materials $2019 ; 12: 2749$.

19. TangT, Liao L., Huang Z., Gu X., and Zhang, X. Accuracy of the evaluation of implant position using a completely digital registration method compared with a radiographic method. J Prosth Dent 2019;122: 537-42.

20. Vercruyssen, Marjolein, Catherine Cox, Ignace Naert, Reinhilde Jacobs, Wim Teughels, and Marc Quirynen. Accuracy and Patient-centered Outcome Variables in Guided Implant Surgery: A RCT Comparing Immediate with Delayed Loading. J Clini Oral Impl Res 2016;27: 427-32.

21. French D, Grandin HM and Ofec R. Retrospective cohort study of 4,591 dental implants: Analysis of risk indicators for bone loss and prevalence of peri-implant mucositis and peri-implantitis. J Periodontol. 2019; 90: 691- 700.

22. Gupta S, Patil N, Solanki J, Singh R and Laller S. Oral Implant Imaging: A Review. Malays J Med Sci. 2015;22:7-17.

23. Fobbe, H, Rammelsberg, P, Lorenzo Bermejo, J, Kappel, S. The Up-to-11-year Survival and Success of Implants and Abutment Teeth under Solely Implant-supported and Combined Tooth-implant-supported Double Crown-retained Removable Dentures. J Clin Oral Impl Res2019; 30:1134-1141.

24. Bernard L, Vercruyssen M, Duyck J, Jacobs R, Teughels $\mathrm{W}$ and Quirynen M. A randomized controlled clinical trial comparing guided with nonguided implant placement: A 3-year follow-up of implant-centered outcomes. J of Prosth Dent 2019; 121: 904-10.

25. Marra R, Acocella A, Rispoli A, Sacco R,Ganz SD and Blasi A. Full-mouth rehabilitation with immediate loading of implants inserted with computer-guided flap-less surgery: a 3-year multicenter clinical evaluation with oral health impact profile. J Impl Dent 2013;22:444-52.

26. Komiyama A, Hultin M, Näsström K, Benchimol D, Klinge B. Soft Tissue Conditions and Marginal Bone Changes around Immediately Loaded Implants Inserted in Edentate Jaws Following Computer Guided Treatment Planning and Flapless Surgery. Clin Oral Implants Res 2012; 14:157-169 